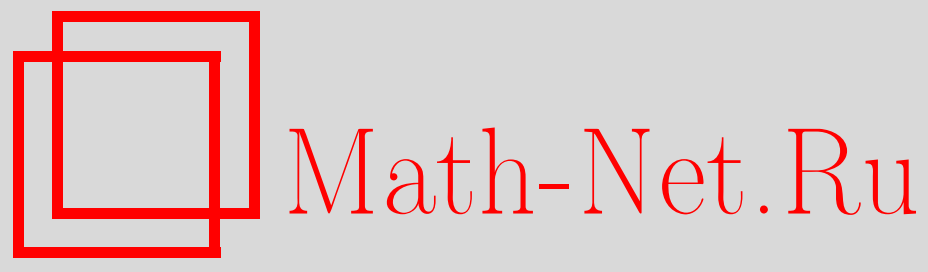

А. Х. Табаров, О некоторых многообразиях абелевых квазигрупп, Дискрет. матем., 2000, том 12, выпуск 3, 154-159

DOI: https://doi.org/10.4213/dm346

Использование Общероссийского математического портала Math-Net.Ru подразумевает, что вы прочитали и согласны с пользовательским соглашением http://www . mathnet.ru/rus/agreement

Параметры загрузки:

IP : 54.166 .219 .16

26 апреля 2023 г., 04:06:14

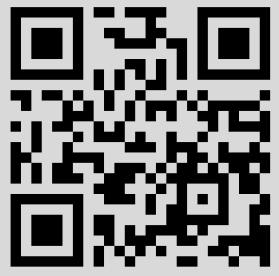


УДК 512.548

\title{
О некоторых многообразиях абелевых квазигрупп
}

\author{
(C) 2000 г. $\quad$ A. X. Табаров
}

\begin{abstract}
Известно, что все примитивные квазигруппы, изотопные группам, характеризуются одним тождеством. Ранее Г. Б. Белявской и автором были описаны многообразия всех примитивных линейных, алинейных и абелевых квазигрупп ( $T$-квазигрупп). В этой работе описываются многообразия абелевых квазигрупп определенного вида.
\end{abstract}

Известно, что все примитивные квазигруппы, изотопные группам, характеризуются одним тождеством (см. [1]). Г. Б. Белявской и автором в [2, 3] были описаны многообразия всех примитивных линейных, алинейных и абелевых квазигрупп ( $T$ квазигрупп). В этой работе описываются многообразия абелевых квазигрупп определенного вида. Заметим, что совпадение класса примитивных абелевых (в смысле Маккензи) квазигрупп с классом $T$-квазигрупп доказано в [4].

Приведем основные определения и сведения, необходимые нам в дальнейшем. Согласно [5], квазигруппа $(Q, \cdot)$ - это множество $Q$ с одной обозначаемой точкой бинарной операцией такой, что каждое из уравнений $a x=b$ и $y a=b$ однозначно разрешимо для любых $a, b \in Q$.

Примитивная квазигруппа $(Q, \cdot, /, \backslash)$ определяется как множество $Q$ с тремя бинарными операциями, связанными соотношениями

$$
(x / y) y=x, \quad x y / y=x, \quad x(x \backslash y)=y, \quad x \backslash x y=y .
$$

Каждой квазигруппе $(Q, \cdot)$ соответствует примитивная квазигруппа $(Q, \cdot, /, \backslash)$, где $(Q, /)$ и $(Q, \bigvee)$ - соответственно правая и левая обратные квазигруппы для $(Q, \cdot)$ :

$$
x y=z \Longleftrightarrow z / y=x \Longleftrightarrow x \backslash z=y .
$$

Квазигруппа $(Q, \circ)$ изотопна квазигруппе $(Q, \cdot)$, если существует такая тройка подстановок $T=(\alpha, \beta, \gamma)$, что

$$
\gamma(x y)=\alpha x \cdot \beta y
$$

для всех $x, y \in Q$.

Квазигруппа $(Q, \cdot)$ называется линейной над группой $(Q,+)($ см. [1]), если $(Q, \cdot)$ изотопна группе $(Q,+)$ и изотопия имеет вид

$$
x y=\varphi x+c+\psi y
$$


где $\varphi, \psi \in \operatorname{Aut}(Q,+), c \in Q$. Частными случаями линейных квазигрупп являются абелевы квазигруппы или $T$-квазигруппы [6] (для них $(Q,+)$ - абелева группа).

Квазиавтоморфизм (антиквазиавтоморфизм) квазигруппы $(Q, \cdot)$ - это главная компонента $\gamma$ автотопии (антиавтотопии) $T(\alpha, \beta, \gamma)$ квазигруппы $(Q, \cdot)$, то есть

$$
\gamma(x y)=\alpha x \cdot \beta y \quad(\gamma(x y)=\alpha y \cdot \beta x)
$$

Согласно лемме 2.5 из [5], любой квазиавтоморфизм группы $(Q,+)$ имеет вид

$$
\gamma x=R_{s} \gamma_{0} x=L_{s} \gamma_{0}^{\prime} x
$$

где $\gamma_{0}, \gamma_{0}^{\prime} \in \operatorname{Aut}(Q,+), R_{s} x=x+s, L_{s} x=s+x$.

Как отмечено в [1], утверждение, аналогичное лемме 2.5 из [5], справедливо и для антиквазиавтоморфизма $\gamma$ (в [1] он называется обратным квазиавтоморфизмом). В этом случае $\gamma_{0}$ и $\gamma_{0}^{\prime}$ из (1) являются антиавтоморфизмами группы $(Q,+)$.

Теорема 1. Для квазигруппы $(Q, \cdot)$ следующие условия эквивалентны:

(1) $(Q, \cdot)$ - абелева квазигруппа, $x y=\varphi x+c+\psi y u \varphi \psi \varphi^{-1}=\psi \varphi^{-1} \psi$;

(2) в примитивной квазигруппе $(Q, \cdot, /, \backslash)$ выполняется тождество

$$
[x(y / u)] z=[x(u / u)](z y / u)
$$

(3) в примитивной хвазигруппе $(Q, \cdot, /, \backslash)$ выполняется тожсдество

$$
x[(u \backslash y) z]=(u \backslash y x)[u \backslash u) z]
$$

Доказательство. Докажем, что из 1 следует 2. Пусть $(Q, \cdot)$ - абелева квазигруппа с условием

$$
\varphi \psi \varphi^{-1}=\psi \varphi^{-1} \psi
$$

Проверим справедливость тождества (2). Заметим, что из равенства $x y=\varphi x+c+\psi y$ следует, что

$$
\begin{aligned}
x / y & =\varphi^{-1} x-\varphi^{-1} c-\varphi^{-1} \psi y, \\
x \backslash y & =-\psi^{-1} \varphi x-\psi^{-1} c+\psi^{-1} y .
\end{aligned}
$$

Тогда

$$
\begin{aligned}
{[x(y / x)] z } & =\varphi[x(y / u)]+c+\psi z \\
& =\varphi\left[\varphi x+c+\psi\left(\varphi^{-1} y-\varphi^{-1} c-\varphi^{-1} \psi u\right)\right]+c+\psi z \\
& =\varphi\left[\varphi x+c+\psi \varphi^{-1} y-\psi \varphi^{-1} c-\psi \varphi^{-1} \psi u\right]+c+\varphi z \\
& =\varphi^{2} x+\varphi c+\varphi \psi \varphi^{-1} y \varphi \psi \varphi^{-1} c \varphi \psi \varphi^{-1} \psi u+c+\psi z
\end{aligned}
$$




$$
\begin{aligned}
{[x(u / u)] \cdot(z y / u)=} & \varphi[x(u / u)]+c+\psi(z y / u) \\
= & \varphi[\varphi x+c+\psi(u / u)]+c+\psi(z y / u) \\
= & \varphi\left[\varphi x+c+\psi\left(\varphi^{-1} u-\varphi^{-1} c-\varphi^{-1} \psi u\right)\right] \\
& \quad+c+\psi\left(\varphi^{-1}(z y)-\varphi^{-1} c-\varphi^{-1} \psi u\right) \\
= & \varphi\left[\varphi x+c+\psi \varphi^{-1} u-\psi \varphi^{-1} c \psi \varphi^{-1} \psi u\right] \\
& \quad+c+\psi \varphi^{-1}(z y)-\psi \varphi^{-1} c-\psi \varphi^{-1} \psi u \\
= & \varphi^{2} x+\varphi c+\varphi \psi \varphi^{-1} u-\varphi \psi \varphi^{-1} c-\varphi \psi \varphi^{-1} \psi u \\
& \quad+c+\psi \varphi^{-1}(\varphi z+c+\psi y)-\psi \varphi^{-1} c-\varphi \psi \varphi^{-1} u \\
= & \varphi^{2} x+\varphi c-\varphi \psi \varphi^{-1} c-\varphi \psi \varphi^{-1} \psi u \\
& \quad+c+\psi z+\psi \varphi^{-1} c+\psi \varphi^{-1} \psi y-\psi \varphi^{-1} c \\
= & \varphi^{2} x+\varphi c+\varphi \psi \varphi^{-1} y-\varphi \psi \varphi^{-1} c-\varphi \psi \varphi^{-1} \psi u+c+\psi z .
\end{aligned}
$$

Следовательно, тождество (2) верно.

Докажем теперь, что из 2 следует 1 . Пусть в примитивной квазигруппе $(Q, \cdot, /, \backslash)$ выполняется тождество (2). Фиксируем в (2) переменную $u$. Тогда по теореме Белоусова о четырех квазигруппах, связанных ассоциативным законом (см. [1]), квазигруппа $(Q, \cdot)$ изотопна группе, поэтому по теоеме Алберта любая лупа, изотопная квазигруппе $(Q, \cdot)$, является группой, в частности, группой является лупа $(Q,+)$, где

$$
x+y=R_{a}^{-1} x \cdot L_{b}^{-1}, \quad a, b \in Q .
$$

Перейдем в (2) к операции + , тогда

$$
\left.R_{a}^{[} R_{a} x+L_{b}(y / u)\right]+L_{b} z=R_{a}\left[R_{a} x+L_{b}(u / u)\right]+L_{b}\left(\left(R_{a} z+L_{b} y\right) / u\right) .
$$

Заменяя $x$ на $R_{a}^{-1} x, z$ на $L_{b}^{-1} z$ и $y$ на $R_{u} y$, находим, что

$$
R_{a}\left[x+L_{b} y\right]+z=R_{a}\left[x+L_{b}(u / u)\right]+L_{b} R_{u}^{-1}\left(R_{a} L_{b}^{-1}+L_{b} R_{u} y\right) .
$$

Полагая в (4) $z=0$ и учитывая, что $u$ - фиксированный элемент, получаем, что

$$
R_{a}\left(x+L_{b} y\right)=\alpha x+\beta y
$$

для соответствующих подстановок $\alpha$ и $\beta$, где $R_{a}$ - квазиавтоморфизм группы $(Q,+)$ :

$$
R_{a} x=\varphi x+k, \quad \varphi \in \operatorname{Aut}(Q,+), \quad k \in Q
$$

Положим в (4) $u=a$ и подберем $x$ так, чтобы $R_{a}\left(x+L_{b}(a / a)\right)=0$. Тогда

$$
\alpha_{1} y+z=L_{b} R_{a}^{-1}\left(R_{a} L_{b}^{-1} z+L_{b} R_{a} y\right)
$$

и $L_{b} R_{a}^{-1}$ - антиквазиавтоморфизм группы $(Q,+)$. Но $R_{a}-$ квазиавтоморфизм, поэтому $L_{b}$ - антиквазиавтоморфизм: $L_{b} y=t+\bar{\psi} y$, где $\bar{\psi}-$ антиавтоморфизм группы $(Q,+), t \in Q$.

Таким образом,

$$
x y=R_{a} x+L_{b} y=\varphi x+c+\bar{\psi} y, \quad c=k+t .
$$


Заметим, что тождество (2) можно записать в виде

$$
x y \cdot z=x(u / u) \cdot[(z \cdot y u / u] .
$$

Прежде чем перейти в (5) к операции + , заметим, что из равенства $x y=\varphi x+c+\bar{\psi}$ следует, что $x / y=\varphi^{-1} x-\varphi^{-1} \bar{\psi} y-\varphi^{-1} c$. Действительно, пусть $x y=z$. Тогда

$$
\begin{aligned}
x & =z / y, \quad z=\varphi^{-1}(z / y)+c+\bar{\psi} y, \\
\varphi^{-1}(z / y) & =z-\bar{\psi} y-c, \quad z / y=\varphi^{-1} z-\varphi^{-1} \bar{\psi} y-\varphi^{-1} c .
\end{aligned}
$$

Следовательно,

$$
\begin{aligned}
x y \cdot z= & \varphi(\varphi x+c+\bar{\psi} y)+c+\bar{\psi} z=\varphi^{2} x+\varphi c+\varphi \bar{\psi} y+c+\bar{\psi} z \\
x(u / u)[(z \cdot y u) / u]= & \varphi(\varphi x+c+\bar{\psi}(u / u))+c+\bar{\psi}[(z \cdot y u) / u] \\
= & \varphi^{2} x+\varphi c+\varphi \bar{\psi}\left(\varphi^{-1} u-\varphi^{-1} \bar{\psi} u-\varphi^{-1} c\right)+c \\
& \quad+\bar{\psi}\left[\varphi^{-1}\left(\varphi z+c+\bar{\psi}(\varphi y+c+\bar{\psi} u)-\varphi^{-1} \bar{\psi} u-\varphi^{-1} c\right]\right. \\
= & \varphi^{2} x+\varphi c-\varphi \bar{\psi} \varphi^{-1} c-\varphi \bar{\psi} \varphi^{-1} \bar{\psi} u+\varphi \bar{\psi} \varphi^{-1} u+c \\
& \quad+\bar{\psi}\left[z+\varphi^{-1} c+\varphi^{-1} \bar{\psi}^{2} u+\varphi^{-1} \bar{\psi} c+\varphi^{-1} \bar{\psi} \varphi y-\varphi^{-1} \bar{\psi} u-\varphi^{-1} c\right] \\
= & \varphi^{2} x+\varphi c-\varphi \bar{\psi} \varphi^{-1} c-\varphi \bar{\psi} \varphi^{-1} \bar{\psi} u \\
& +\varphi \bar{\psi} \varphi^{-1} u+c-\bar{\psi} \varphi^{-1} c-\bar{\psi} \varphi^{-1} \bar{\psi} u \\
& +\bar{\psi} \varphi^{-1} \bar{\psi} \varphi y+\bar{\psi} \varphi^{-1} \bar{\psi}^{2} u+\bar{\psi} \varphi^{-1} c+\bar{\psi} z .
\end{aligned}
$$

Отсюда следует, что

$$
\begin{array}{rl}
\varphi^{2} x+\varphi c+\varphi \bar{\psi} y+c+\bar{\psi} z=\varphi^{2} & x+\varphi c-\varphi \bar{\psi} \varphi^{-1} \\
& -\varphi \bar{\psi} \varphi^{-1} \bar{\psi} u+\varphi \bar{\psi} \varphi^{-1} u+c-\bar{\psi} \varphi^{-1} c \\
& -\bar{\psi} \varphi^{-1} \bar{\psi} u+\bar{\psi} \varphi^{-1} \bar{\psi} \varphi y+\bar{\psi} \varphi^{-1} \bar{\psi} c \\
& +\bar{\psi} \varphi^{-1} \bar{\psi}^{2} u+\bar{\psi} \varphi^{-1} c+\bar{\psi} z .
\end{array}
$$

После сокращения получаем, что

$$
\begin{aligned}
\varphi \bar{\psi} y+c=\varphi \bar{\psi} \varphi^{-1} c-\varphi \bar{\psi} \varphi^{-1} \bar{\psi} u+\varphi \bar{\psi} \varphi^{-1} u+c-\bar{\psi} \varphi^{-1} c-\bar{\psi} \varphi^{-1} \bar{\psi} u \\
+\bar{\psi} \varphi^{-1} \bar{\psi} \varphi y+\bar{\psi} \varphi^{-1} \bar{\psi} c+\bar{\psi} \varphi^{-1} \bar{\psi}^{2} u+\bar{\psi} \varphi^{-1} c
\end{aligned}
$$

Пусть $u=0$. Тогда

$$
\varphi \bar{\psi} y+c=-\varphi \bar{\psi} \varphi^{-1} c+c-\bar{\psi} \varphi^{-1} c \bar{\psi} \varphi^{-1} \bar{\psi} \varphi y+\bar{\psi} \varphi^{-1} \bar{\psi} c+\bar{\psi} \varphi^{-1} c
$$

Полагая $y=0$, находим, что

$$
c=-\varphi \bar{\psi} \varphi^{-1} c+c-\bar{\psi} \varphi^{-1} c+\bar{\psi} \varphi^{-1} \bar{\psi} c+\bar{\psi} \varphi^{-1} c .
$$

Подставляя это выражение для $c$ в левую часть предыдущего равенства, находим, что

$$
\begin{aligned}
\varphi \bar{\psi} y-\varphi \bar{\psi} \varphi^{-1} c+c-\bar{\psi} \varphi^{-1} \bar{\psi} c+\bar{\psi} \varphi^{-1} \bar{\psi} c+\bar{\psi} \varphi^{-1} c \\
=-\varphi \bar{\psi} \varphi^{-1} c+c-\bar{\psi} \varphi^{-1} c+\bar{\psi} \varphi^{-1} \bar{\psi} \varphi y+\bar{\psi} \varphi^{-1} \bar{\psi} c+\bar{\psi} \varphi^{-1} c
\end{aligned}
$$


откуда после сокращений приходим к равенству

$$
\varphi \bar{\psi} y-\varphi \bar{\psi} \varphi^{-1} c+c-\bar{\psi} \varphi^{-1} c=-\varphi \bar{\psi} \varphi^{-1} c+c-\bar{\psi} \varphi^{-1} c+\bar{\psi} \varphi^{-1} \bar{\psi} \varphi y .
$$

Положим $-\varphi \bar{\psi} \varphi^{-1} c+c-\bar{\psi} \varphi^{-1} c=p$, где $p \in Q$. Тогда

$$
\varphi \bar{\psi} y+p=p+\bar{\psi} \varphi^{-1} \bar{\psi} \varphi y
$$

или

$$
\tilde{R}_{p} \varphi \bar{\psi} y=\tilde{L}_{p} \bar{\psi} \varphi^{-1} \bar{\psi} \varphi y
$$

откуда

$$
\tilde{L}_{p}^{-1} \tilde{R}_{p} y=\bar{\psi} \varphi \bar{\psi} \varphi^{-1} y .
$$

Но $\bar{\psi} \varphi \bar{\psi} \varphi^{-1}$ - антиавтоморфизм группы $(Q,+)$. Поэтому

$$
\tilde{L}_{p}^{-1} \tilde{R}_{p}(x+y)=\tilde{L}_{p}^{-1} \tilde{R}_{p} y+\tilde{L}_{p}^{-1} \tilde{R}_{p} x
$$

или

$$
-p+x+y+p=-p+y+p-p+x+p,
$$

откуда $x+y=y+x$, то есть группа $(Q,+)$ абелева и поэтому $\bar{\psi}=\psi \in \operatorname{Aut}(Q,+)$. Следовательно, $(Q, \cdot)$ - абелева полугруппа. Далее, в абелевой группе $\tilde{L}_{x}=\widetilde{R}_{x}$ для любого $x \in Q$. Поэтому из (6) следует, что $\varphi \psi y=\psi \varphi^{-1} \psi \varphi y$ или $\varphi \psi \varphi^{-1}=\psi \varphi^{-1} \psi$, что и требовалось доказать.

Импликации $1 \Rightarrow 3$ и $3 \Rightarrow 1$ проверяются аналогичным образом.

Следствие 1. Многообразие всех примитивных абелевых квазигрупп с условием $\varphi \psi \varphi^{-1}=\psi \varphi^{-1} \psi$ характеризуется тождеством (2) или (3).

Приведем другие условия на автоморфизмы, приводящие к некоторым подмногообразиям абелевых квазигрупп, связанным с хорошо известными (неуравновешенными) тождествами.

Теорема 2. Для абелевой хвазигруппы $(Q, \cdot)$ следующие условия эквивалентны:

(1) квазигруппа $(Q, \cdot)$ имеет вид

$$
x y=\varphi x+\psi y
$$

əде $\varphi^{2}+\psi=\psi^{2}+\varphi=\varphi \psi, c=0$

(2) в $(Q, \cdot)$ выполняется второе тождество Стейна

$$
x \cdot y x=y x \cdot y
$$

Доказательство. Докажем, что $2 \Rightarrow 1$. Пусть в абелевой квазигруппе $(Q, \cdot)$ выполняется тождество $x \cdot y x=y x \cdot y$. Тогда $(Q, \cdot)$ идемпотентна (см. [8]), следовательно, медиальна (см. [6]) и $c=0, \varphi+\psi=\varepsilon$. Учитывая это, переходим в тождестве $x \cdot y x=y x \cdot y$ к операции + и получаем, что

$$
\begin{aligned}
& x \cdot y x=\varphi x+\psi(\varphi y+\psi x)=\varphi x+\psi \varphi y+\psi^{2} x \\
& y x \cdot y=\varphi(\varphi y+\psi x)+\psi y=\varphi^{2} y+\varphi \psi x+\psi y
\end{aligned}
$$


или

$$
\varphi x+\psi \varphi y+\psi^{2} x=\varphi^{2} y+\varphi \psi x+\psi y \text {. }
$$

Пусть $x=0$, тогда $\psi \varphi y=\varphi^{2} y+\psi y, \psi \varphi=\varphi^{2}+\psi$. Полагая в (7) $y=0$, находим, что

$$
\varphi x+\psi^{2} x=\varphi \psi x, \quad \varphi \psi=\psi^{2}+\varphi
$$

В силу медиальности $(Q, \cdot)$

$$
\varphi^{2}+\psi=\psi^{2}+\varphi=\varphi \psi
$$

Обратная импликация легко проверяется.

Аналогично можно доказать, что для абелевых квазигрупп справедливо следующее утверждение.

Теорема 3. Для абелевой квазигруппы $(Q, \cdot)$ следующие условия эквивалентны:

(1) квазигруппа $(Q, \cdot)$ имеет вид

$$
x y=\varphi x+\psi y
$$

где $\psi=\varphi+\varepsilon,(Q, \cdot)-$ әруппа эхспонента два

$$
\left(x y=\varphi x+c+\psi y, \quad \varphi \psi=J \psi \varphi, \quad \varphi^{2}+\psi^{2}=\varepsilon, \quad \varphi c+\psi c+c=0\right)
$$

(2) в квазигруппе $(Q, \cdot)$ выполняется первое (второе) тождество Шредера

$$
x \cdot x y=x y \cdot y \quad(x y \cdot y x=x) .
$$

\section{Список литературы}

1. Белоусов В. Д., Уравновешенные тождества в квазигруппах. Матем. сб. (1966) 70, 5597.

2. Belyavskaya G., Tabarov A., Characterization of primitive linear, alinear, and abelian quasigroups. Quasigroups and Related Systems (1994) 1, №1, 8-21.

3. Белявская Г. Б., Табаров А. Х., Характеристика линейных и алинейных квазигрупп. Дискретная математиха (1992) 2, №2, 142-147.

4. Belyavskaya G. B., Abelian quasigroups are T-quasigroups. Quasigroups and Related Systems (1994) 1, №1, 39-49.

5. Белоусов В. Д., Основъ теории квазигрупп и луп. Наука, Москва, 1967.

6. Kepka T., Nemec P., T-quasigroups. Acta Univ. Carolinae Math. \& Phys. (1971) 12, №1, 39-49, №2, 31-49.

7. Табаров А. Х., Т-квазигруппь с дополнительнъми тождествами. Деп. ВИНИТИ 09.01.91, №163-B91.

8. Белоусов В. Д., Парастрофно-ортогоналъные квазигруппъ. Штиинца, Кишинев, 1983.

Статья поступила 12.02.2000. 